\title{
Biham-Middleton-Levine Traffic Model in Two-Dimensional Hexagonal Lattice
}

\author{
J. Carlos García Vázquez, Salvador Rodríguez Gómez, \\ and Fernando Sancho Caparrini
}

\begin{abstract}
In this paper, a Biham-Middleton-Levine traffic model on a 2D hexagonal lattice is studied by computer simulations and its behavior is compared with the BML model on a 2D square lattice and a 3D cubic lattice (Supported by Excellence project TIC-6064 of Junta de Andalucía cofinanced with FEDER founds).
\end{abstract}

\subsection{Introduction}

In [1] Biham, Middleton and Levine (BML) introduced one of the most studied models about traffic flow in recent years. Based on a two-dimensional cellular automaton, this extremely simple model exhibits self-organization, pattern formation and phase transitions.

BML model considers the motions of two species of particles, north- and eastbound particles, in a two dimensional square lattice with $N \times N$ sites with periodic boundary conditions in such a way that the geometric model corresponds to the discrete torus $\mathbb{Z}_{N} \times \mathbb{Z}_{N}$. Several interesting generalizations of the model have been considered, such as free boundary conditions [6], non-square aspect ratio of the underlying square lattice [5], four-directional traffic [3], and an extension to three dimensional cubic lattice with periodic boundary conditions [2].

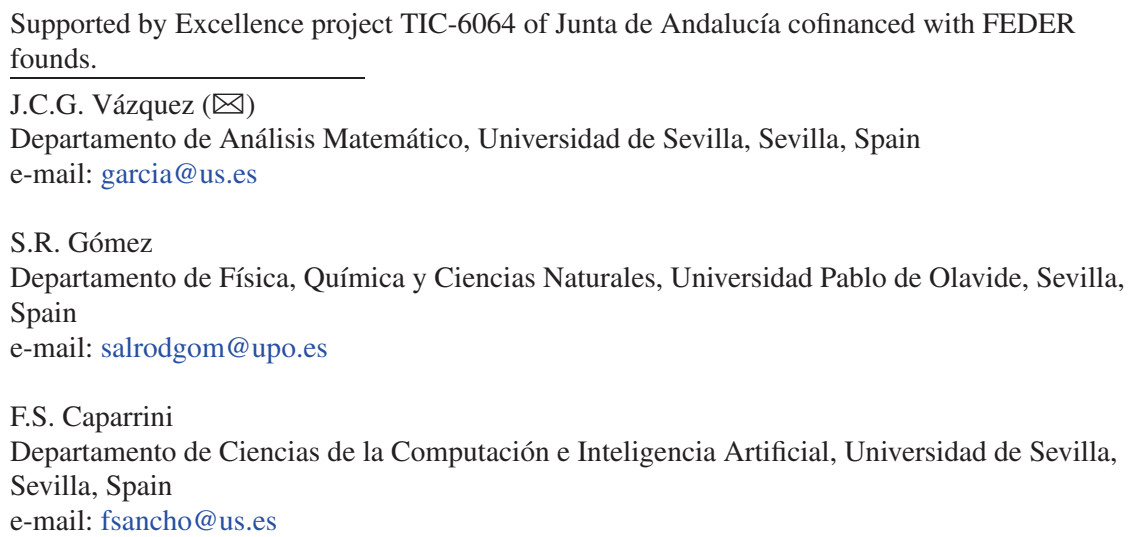


Fig. 113.1 BML model on a

two dimensional hexagonal

tesselation with periodic

boundary conditions

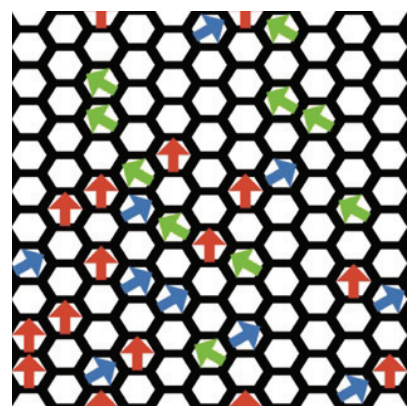

In this paper we consider a BML model on a two-dimensional hexagonal lattice and we pose the question of compare this model with the 2D square lattice [1] and the $3 \mathrm{D}$ cubic lattice case [2].

\subsection{BML on a Two-Dimensional Hexagonal Lattice}

We consider an hexagonal lattice tessellation of the torus with three families of particles (red, blue and green) that try to move in north, north-east and north-west direction. In order to consider the hexagonal lattice with periodic boundary conditions we need the system size be an even natural number (see Fig. 113.1).

Initially, particles are placed randomly according to a parameter $0 \leq p \leq 1$ which is the probability of a lattice site to contain a particle. Each hexagonal cell has a probability $p / 3$ of having a blue particle, $p / 3$ of having a red particle, $p / 3$ of having a green particle and $1-p$ of being empty. In this way $p$ is the particle density in the system.

The discrete time dynamics is determined by the following rules: (a) first, all red particle synchronously try to move forward one site in north direction. If the site northward of a red particle is currently empty, it advances. Otherwise that red particle stays in the present location, even if the northward site is to become empty during the current time step; (b) second, blue particles follow the same rules but they try to move in north-east direction; (c) finally, green particles try to move in north-west direction following the same rules as before. This marks the end of a time step and the above particle moving process is repeated over and over again. Let us observe that the dynamics is fully deterministic and the only random event in the model occurs in the initial condition.

In every discrete time step $t$, the instantaneous speed of the system $v_{t}$ is computed as the ratio between the number of particles that succeed to move and the total number of them. If $v_{t}=0$ then no particle has moved in the time step $t$; if $v_{t}=1$ then all particles have moved. The asymptotic speed $v$ is the speed $v_{t}$ averaged over the asymptotic cycle and $\langle v\rangle$ is the average asymptotic speed over the random initial configurations. We want to study the average asymptotic speed $\langle v\rangle$ as a function of $p$. 

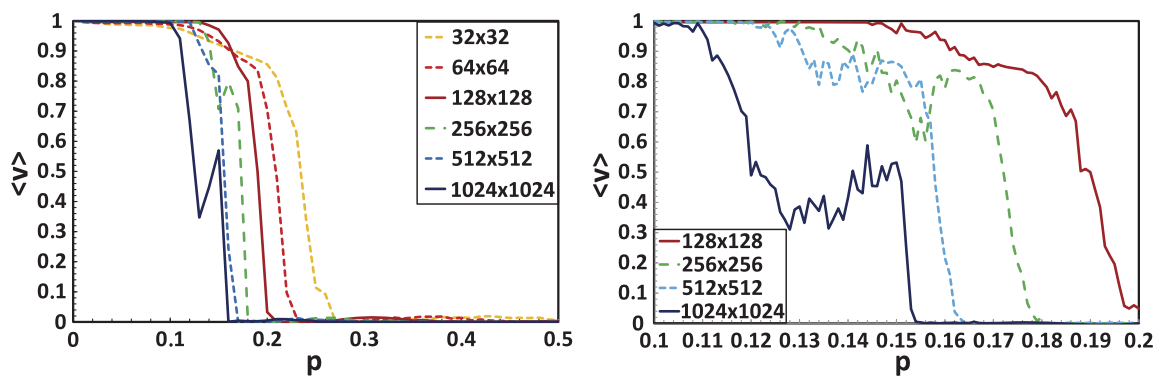

Fig. 113.2 $\langle v\rangle$ as a function of $p$ for different system sizes

\subsection{Simulation Results}

We have implemented the BML model on hexagonal lattices of finite size $N \times N$, for $N=32,64,128,256,512$ and 1024 .

In the left panel of Fig. 113.2, $\langle v\rangle$ is seen as a function of $p$. These curves have been obtained as an average of 1000 random initial configurations for every value of $p$. The asymptotic speed $v$ for every initial configuration is approximated by the average of $v_{t}$ between time steps $t=50.000$ and $t=51.000$. The step increment of the parameter $p$ has been of 0.01 units. Since we have a special interest in the sudden drop of $\langle v\rangle$, we have completed the numerical study repeating the experiments moving $p$ from 0.1 to 0.2 with increments of 0.001 and with the same conditions as before. Results can be seen in the right panel of Fig. 113.2.

\subsection{Analysis of the Results}

Similar to the results in [1] for the 2D square lattice case and to [2] for the 3D cubic lattice case, in the 2D hexagonal lattice case there are two qualitatively different asymptotic states separated by a sharp dynamical transition. Below the transition all particles move freely and the system reaches asymptotic speed 1 or very close to 1 (the free flow phase) and above the transition the particles get stuck and the system gets speed 0 (the global jam phase). It is interesting to observe the geometric patterns of the system. Below the transition the system is able to self-organize and to form very well defined geometric patterns that can be seen in the left panel of Fig. 113.3: ordered monochromatic clusters of particles almost freely flowing. Above the transition the system forms one or more percolating clusters of jammed particles (right panel of Fig. 113.3).

Following [1] we define the critical region as the range of densities where both asymptotic states can be found with a non 0 probability. The size of this region depends on the system size and the critical probability is taken as the center of the critical region. We have made an estimation of the value of the critical probability for different system sizes. We have considered the critical region as the region of 
Fig. 113.3 Typical geometric patterns of the free flow and global jam phases in the hexagonal lattice BML model

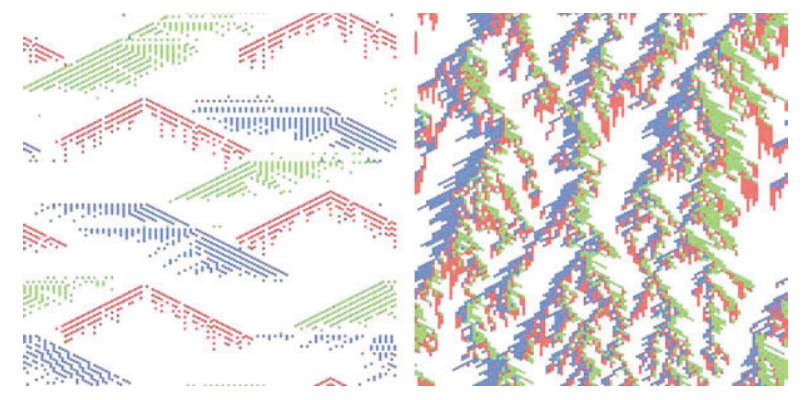

Table 113.1 Critical region as a function of $N$

\begin{tabular}{llll}
\hline$N$ & $\langle v\rangle<0.99$ & $\langle v\rangle>0.01$ & Critical density \\
\hline 128 & $p>0.14$ & $p<0.2$ & 0.17 \\
256 & $p>0.13$ & $p<0.18$ & 0.155 \\
512 & $p>0.12$ & $p<0.17$ & 0.145 \\
1024 & $p>0.1$ & $p<0.16$ & 0.13 \\
\hline
\end{tabular}

densities for which speed lies between 0.01 and 0.99 , and the critical probability as the center of the interval. We see, as in [1], that the critical probability slightly decrease as the system size increases (see Table 113.1).

In [1] Biham, Middleton and Levine reported the existence of two phases in the two dimensional square lattice model separated by a sharp dynamical transition, and the general belief was that the system showed a first order transition. However, D'Souza $[4,5]$ found a region of metastable intermediate states with an asymptotic speed around $v=2 / 3$, and with a very well defined geometry. Thus, the BML model does not necessarily exhibit a sharp phase transition from free flow to global jam, but instead has a range of intermediate states with regions of free flow intersecting at jammed wave-fronts. In the 2D hexagonal lattice case, as in the 3D cubic lattice case, we report that we have not found intermediate states: asymptotic speed is always very close to 1 or to 0 . Although we have observed a few results with $v$ around 0.84 the number of that cases is decreasing when the system size grows.

The curves for the hexagonal lattice model are more similar to the 3D cubic lattice than to the 2D square lattice case. From the results we believe that the BML model on the hexagonal lattice may show a first order phase transition. To support this hypothesis we have plotted the hysteresis curve (Fig. 113.4) obtained in the following way: starting from $p=0$, the density is increased by a small increment by randomly introducing particles to the empty sites of the system. Then the system evolves until it reaches a recurrent state and speed is measured. This is done again and again until $p=1$. The blue curve represents this evolution. Now, we decrease the density by the same increment by removing randomly particles from the system, following the same process. The red line represents this process. In this way the hysteresis loop, typical of first order phase transitions, is obtained. 
Fig. 113.4 Hysteresis curve: the blue and red curves represent the evolution of the system when particles are slowly added to or removed from the system, respectively

Fig. 113.5 Typical geometric pattern of the slow speed phase in the hexagonal lattice BML model
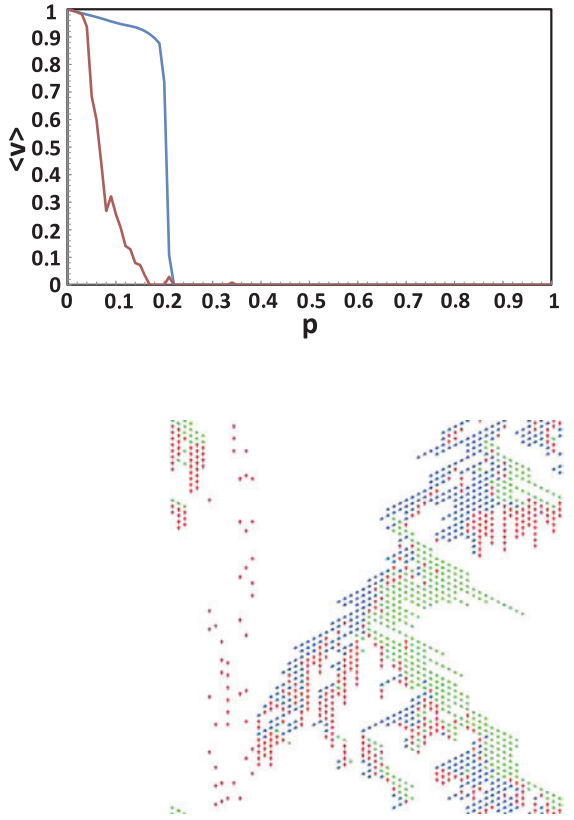

In [2] the authors also point out one interesting difference with respect the 2D square lattice case. Besides the free flow phase and the global jam phase, they observed an extensive region of densities with a low but non 0 asymptotic speed. According to the authors this phase is a result of the formation of spatially limitedextended percolating cluster of particles, where most particles are jammed by colliding into the percolating cluster and a small number of residual particles freely move. Since almost all particles are jammed the speed is very slow. This is a difference with the 2D square lattice case, because in that case all particles will eventually merge into the percolating backbone leading to a completely jamming configuration. Completely similar to the 3D cubic lattice case, in the 2D hexagonal lattice case there exists a slow speed phase where speed is very close to 0 , but greater than 0 . The system is able to form percolating clusters of jammed particles letting a residual small number of particles flow freely. In Fig. 113.5 we can see a typical geometric pattern of this phase.

One of the complications of the BML model from a theoretical point of view is that it is not a monotonous model in the following sense: adding particles to a configuration that is known to jam can actually change the sequence of particle interactions and result a configuration going to free flowing instead of jamming. In this model we can see this kind of behavior, where increasing the density causes increased speed. This can be seen clearly in the critical region for system sizes 256 and 1024 (Fig. 113.2) and to a lesser extent in the slow speed phase, where we can see a peak in the plots (Fig. 113.6). Our model can be a tool to study this behavior that does not appear in the other two models. 
Fig. 113.6 $\langle v\rangle$ is not decreasing as a function of $p$ in the slow speed phase

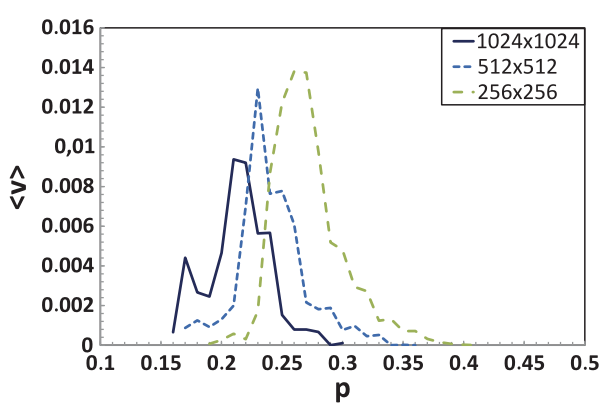

\subsection{Conclusions}

From the numerical results we conclude that the phase diagram of the $2 \mathrm{D}$ hexagonal lattice model is more similar to the $3 \mathrm{D}$ cubic lattice than to the $2 \mathrm{D}$ square lattice case. We can reproduce most of the features of the $3 \mathrm{D}$ cube lattice case which do not appear in the 2D square lattice case, by changing the topology of the lattice that supports the 2D model.

\section{References}

1. Biham O, Middleton AA, Levine D (1992) Self organization and a dynamical transition in traffic flow models. Phys Rev A 46:6124-6127

2. Chau HF, Wan KY (1999) Phase diagram of the Biham-Middleton-Levine traffic model in three dimensions. Phys Rev E 60:5301-5304

3. Huang D, Huang W (2006) Biham-Midleton-Levine model with four-directional traffic. Physica A $370: 747-755$

4. Linesch NJ, D'Souza RM (2008) Periodic states, local effects and coexistence in the BML traffic jam model. Physica A 387:6170-6176

5. D'Souza RM (2005) Coexisting phases and lattice dependence of a cellular automata model for traffic flow. Phys Rev E 71:066112

6. Tadaki S (1994) Two dimensional cellular automaton model of traffic flow with open boundaries. Phys Rev E 54:2409-2413 